\title{
Efecto del menú balanceado en usuarios de servicios de alimentación empresarial
}

\section{The effect of a balanced menu on company food-service users}

\author{
Diana M. Orozco-Soto y Lilliana M. Troncoso-Piedrahita
}

Diana María Orozco-Soto, Msc., Nutricionista Dietista, profesora Escuela de Nutrición y Dietética, Universidad de Antioquia, Medellín, Colombia, correo electrónico dimaorso@pijaos.udea.edu.co Lilliana María Troncoso Piedrahita, Msc. Esp., Nutricionista Dietista, profesora Escuela de Nutrición y Dietética, Universidad de Antioquia, Medellín, Colombia, correo electrónico lilianat@pijaos.udea.edu.co

Recibido 9 Septiembre 2010/Enviado para Modificación 25 Mayo 2011/Aceptado 12 Julio 2011

\section{RESUMEN}

Objetivo Analizar el efecto del menú servido nutricionalmente balanceado, a empleados de Empresas Publicas de Medellín en los campamentos de generación de energía (Guadalupe, Guatapé-Playas y La Sierra), en el índice de masa corporal (IMC), porcentaje de grasa corporal, niveles de lípidos séricos, después de una intervención de cuatro meses, entre diciembre de 2009 y marzo de 2010.

Métodos Estudio cuasi experimental de intervención a alimentación suministrada en cantidad y calidad a 129 empleados voluntarios, evaluando las variables antes y después, para establecer cambios.

Resultados Después de la intervención los lípidos séricos se redujeron significativamente $(p<0,001)$, específicamente colesterol total $-13,3 \pm 36,4 \mathrm{mg} / \mathrm{dl}$ (IC $95 \%-214 ; 68$ ) y triglicéridos $-81,2 \pm 138,1 \mathrm{mg} / \mathrm{dl}$ (IC $95 \%-757 ; 81$ ); el IMC bajó $0,13 \pm 0,76$ unidades (IC $95 \%-0,3 ; 0,01)$ y el porcentaje de grasa corporal varió $-0,31 \pm 2,19 \%$ (IC $95 \%-0,69 ; 0,07$ ), a pesar de ser reducciones no significativas (IMC: $p=0,63$ y \% de Grasa: $p=0,11$ ) sí son biológicamente plausibles dada su relación con disminución de riesgos de salud.

Conclusión Los servicios de alimentación a colectivos son lugares en los cuales se pueden realizar intervenciones nutricionales efectivas que favorezcan el estado de salud y nutrición de los usuarios atendidos, sin representar complicaciones en la producción.

Palabras Clave: Servicios de alimentación, planificación de menú, dislipidemias, Índice de Masa Corporal (fuente: DeCS, BIREME).

\section{ABSTRACT}

Objetive Analyzing the effect of a nutritionally-balanced menu served to Medellín public company employees (Guadalupe, Guatapé-Playas and La Sierra) on their 
body-mass index (BMI), percentage of body fat and serum lipid levels following a four-month intervention between December 2009 and March 2010.

Methods This involved a quasi-experimental intervention regarding the food supplied to 129 volunteer employees in terms of quantity and quality; variation in variables was evaluated before and after the intervention.

Results Serum lipid levels became significantly reduced following the intervention, $(p<0.001)$, specifically total cholesterol $(13.3 \pm 36.4 \mathrm{mg} / \mathrm{dl} ;-214-6895 \% \mathrm{Cl})$ and triglycerides $(-81.2 \pm 138.1 \mathrm{mg} / \mathrm{dl} ;-757-8195 \% \mathrm{Cl})$. The BMI was down by $0.13 \pm$ 0.76 units $(-0.3-0.0195 \% \mathrm{Cl})$ and $\%$ body fat varied by $-0.31 \pm 2.19 \%(-0.69-0.0795$ $\% \mathrm{Ci}$ ) despite these being statistically non-significant reductions (BMI: $p=0.63 ; \%$ fat: $p=0.11$ ); however, they were biologically plausible because of their relationship to reduced health hazards.

Discussion Canteens are places where effective nutritional interventions can be made to promote their users' health and nutrition without representing service production complications.

Key Words: Food service, menu planning, dyslipidaemia, body mass index (source: $\mathrm{MeSH}, \mathrm{NLM}$ ).

$\mathrm{D}$ e acuerdo con la Organización Mundial de la Salud (OMS) las enfermedades crónicas se constituyen hoy día en la principal causa de muerte en el mundo, específicamente las de tipo cardiovascular, estableciéndose una pandemia de difícil manejo de atención en salud, sin embargo, se ha probado que la adopción de estilos de vida saludables como los relacionados con la alimentación son clave en la prevención de éstas. La situación es compleja en la población adulta pues cada vez las patologías de este tipo aparecen a edades más tempranas, por tanto la labor de prevención debe ser prioridad para todos los organismos y profesionales de salud, tal como lo especifica la OMS en su informe sobre la salud del mundo 2008 (1).

En el área de servicios de alimentación el nutricionista dietista debe tener como pilares permanentes la promoción y la prevención, siendo los servicios de alimentación espacios que privilegian la posibilidad de informar y educar a sobre los beneficios de una alimentación saludable. Un ejemplo de ello ha sido documentado por la Organización Internacional del Trabajo (OIT) en 2005, demostrando que las acciones de intervención en alimentación y nutrición desarrolladas en servicios de alimentación de diferentes empresas en el mundo han dado resultados positivos sobre la salud de trabajadores beneficiarios y las propias organizaciones, enfatizando en cómo estos escenarios se constituyen en emplazamientos idóneos para las acciones de promoción y prevención de la salud, en la medida que atienden colectivos humanos ampliando la cobertura y los resultados de las intervenciones (2). 
En Colombia no se han reportado hasta ahora experiencias similares en servicios de alimentación, pero sí se conoce que las cifras de personas con problemas de sobrepeso, obesidad y enfermedades crónicas va en aumento, y que es precisamente la población adulta la principal afectada, en su mayoría en edad productiva, sin que hasta ahora se hayan logrado mitigar las cifras que hasta el 2005 representaban cerca del 50 \% de la población según la Encuesta Nacional de la Situación Nutricional de Colombia 2005 (ENSIN), a pesar de las diferentes acciones que distintas organizaciones realizan a diario para contribuir al manejo del problema catalogado de salud pública en el Artículo primero de la Ley 1355/09 del Congreso de Colombia (3, 4).

En el caso particular de Empresas Públicas de Medellín (EPM) en sus campamentos de generación de energía ubicados en el Departamento de Antioquia, cerca a los municipios de Guatapé, San Rafael, Guadalupe y Puerto Nare, el personal presentó altos índices de sobrepeso y obesidad visibles, así como enfermedades derivadas de éstas, y en exámenes rutinarios realizados por su Administradora de Riesgos Profesionales (A.R.P.) en el segundo semestre de 2008, se detectó que al menos el $90 \%$ de los trabajadores tenían alterado su perfil lipídico, afectando su salud y calidad de vida, al tiempo que limitaba su productividad laboral medida por el incremento del ausentismo debido a incapacidades y permisos médicos. No obstante, éstos resultados hasta ese momento sólo eran usados para la clasificación epidemiológica de la población, pero no eran objeto de intervención por parte de la A.R.P. o la empresa, ni se había definido una posible causa de las alteraciones. Para el caso de personas con cifras críticas eran remitidas a su entidad prestadora de salud (EPS) para su tratamiento.

Por lo tanto, la empresa identificó la importancia de intervenir la problemática a fin de controlar y prevenir los efectos negativos causados por el alto índice de morbilidad en los empleados asociados a la alimentación no controlada en los campamentos. Por otra parte, la Resolución 3673 de 2008 del Ministerio de la Protección Social establece el reglamento técnico de trabajo seguro en alturas que aplica de forma obligatoria para más del $50 \%$ de éstos trabajadores, en el que se especifica que quienes padezcan "patologías metabólicas, cardiovasculares, que generen vértigo, alteraciones del equilibrio...", no podrán realizar estas tareas, y declara que también será tenido en cuenta el IMC y el peso del trabajador, lo cual obligó de forma simultánea a dar relevancia a esta situación, justificando la aplicación del proyecto para el diagnóstico e intervención de los servicios de alimentación con acompañamiento de profesionales de la Nutrición, que aportaran a la solución del problema y a mejorar su funcionamiento (5). 
Estos trabajadores permanecen en promedio cinco días a la semana en el campamento donde reciben toda la alimentación diaria en los servicios de alimentación que funcionan por autogestión, distribuida en tres comidas principales (desayuno, almuerzo y comida), las cuales al ser evaluadas en el año 2009, en número y tamaño de porciones servidas sobrepasaba la recomendación de calorías y nutrientes para ellos en más de un $30 \%$, puesto que no se regulaba el aporte nutricional de los alimentos servidos en combinación, formas de preparación, cantidad y tamaño de porción, además se ofrecía consumo libre de bebidas gaseosas y café azucarado en diferentes espacios del campamento, aspectos que podrían haber estado relacionado con las alteraciones de sobrepeso y perfil lipídico de la población. La actividad física se evalúo moderada en el trabajo y poca de tipo recreativa, representando un gasto energético menor a lo consumido diariamente.

Como respuesta se planteó analizar el efecto de un menú nutricionalmente balanceado, en el IMC, porcentaje de grasa corporal y niveles de lípidos séricos de los empleados de los campamentos, basados en las experiencias internacionales reportadas por la OIT y las posibilidades del servicio de alimentación para la promoción y prevención por medio de la oferta de menús más saludables.

\section{MATERIALES Y MÉTODOS}

Se realizó un estudio cuasi experimental (6), definido así por las características de la intervención realizada, la imposibilidad de aleatorizar y controlar todas las variables, el tipo de población estudiada y su dinámica cotidiana en la empresa. Se comparan los datos de IMC, \% de grasa corporal y lípidos séricos obtenidos antes y después de los usuarios del servicio de alimentación de la empresa, que voluntariamente se adhirieron al estudio y tuvieron una exposición promedio de cuatro meses a la intervención. Se tuvo como población objetivo empleados que no fueran del área de la salud, practicantes o contratistas, en total 368 empleados se invitaron a participar del estudio. De éstos, cumplieron con los criterios de inclusión (contratación directa e indefinida con EPM, asignados a la central de tiempo completo por más de un año; que al momento del inicio del estudio tuviese alterado el IMC o alguno de los valores de lípidos séricos) y firmaron el consentimiento 129 personas; excluyendo aquellos con patologías de especial cuidado. 
La información diagnóstica y de control nutricional del usuario (datos generales, antropométricos y epidemiológicos), fue tomada en los centros médicos de cada campamento, mediante evaluación individual realizada por nutricionistas dietistas profesionales, quienes tomaron las medidas corporales y los pliegues cutáneos de grasa, siguiendo lo establecido para toma de datos antropométricos según estándares internacionales (7). El porcentaje de grasa se obtuvo con la ecuación de Durning y Womersley considerada la más aproximada para la población evaluada (8). La clasificación de IMC y porcentaje de grasa se adoptó de la OMS 2000 (9). Igualmente la primera evaluación indagó por datos alimentarios de frecuencia de consumo de alimentos por fuera de los servicios de restaurante, específicamente bebidas como café, gaseosas, jugos comerciales, y aromáticas azucaradas, además de dulces, postres y snacks.

Los datos de perfil lipídico (Triglicéridos (TG), Colesterol Total (CT), Colesterol HDL (cHDL) y Colesterol LDL (cLDL)), fue tomada de los resultados de laboratorio reportados trimestralmente por la -A.R.P-. La toma de muestras de sangre fue realizada por personal calificado, en los campamentos y se trasladaron a Medellín para su análisis en el laboratorio que le sirve a la A.R.P de forma rutinaria y bajo estrictos estándares de calidad. La técnica utilizada para la determinación cuantitativa de los lípidos séricos fue el método enzimático colorimétrico $(10,11)$ tomando como puntos de corte los valores de referencia del ATPIII: CT deseado $<200 \mathrm{mg} / \mathrm{dl}$; cHDL normal $>40 \mathrm{mg} / \mathrm{dl}$ en hombres y $>50 \mathrm{mg} / \mathrm{dl}$ en mujeres; cLDL óptimo $<100 \mathrm{mg} / \mathrm{dl}$; y TG normales $<150 \mathrm{mg} / \mathrm{dl}(12)$.

El proyecto fue sometido a consideración del Comité de Bioética de Investigación en Humanos -CBEIH- de la Sede de Investigación Universitaria -SIU- de la Universidad de Antioquia, que avaló su ejecución declarando que éste se ajustaba a las buenas prácticas clínicas y no involucraba riesgos significativos para la población.

La intervención se realizó sobre el menú ofrecido a toda la población, modificado en el tipo y composición de las preparaciones servidas, pero sólo se limitó la cantidad consumida de alimentos a aquellas personas que aceptaron hacer parte del estudio. Para lograr dicha meta fue indispensable que en el servicio de alimentación se realizaran cambios que garantizaran realmente el aporte del menú recomendado para los usuarios. Por ello fue necesario dedicar seis meses de trabajo previo para la preparación del servicio de alimentación, que correspondió a capacitación al personal manipulador de 
alimentos, la implementación de procesos de Buenas Prácticas de Manufactura -BPM-, métodos de cocción adecuados e inclusión de nuevos ingredientes, lo cual estuvo acompañado de información al consumidor, de modo tal que éste se enterara de cuáles eran los cambios que se harían, por qué, y qué beneficios tendría con ellos, para disminuir complicaciones y resistencia de los usuarios al momento cambiar el menú.

Se planeó suministrar con la alimentación 2700 calorías por día, teniendo en cuenta un margen de 300 calorías sobre lo recomendado para el grupo (3 $000 \mathrm{Kcal}$ ), con el objetivo de no generar excesos de consumo calórico en los sujetos, dado que en los intermedios de la mañana y la tarde ellos pueden consumir gaseosas, cafés con azúcar u otras bebidas que tienen disponibles en el campamento y en los puestos de trabajo de forma libre. Las recomendaciones nutricionales fueron tomadas de las tablas vigentes del Instituto Colombiano de Bienestar Familiar -ICBF- (1988), para hombres adultos de 25 a 49 años por ser el grupo más prevalente en la población atendida (13). La distribución del valor calórico total de macro nutrientes se realizó siguiendo las recomendaciones de las guías alimentarias para la población colombiana mayor de dos años, que acatan las sugerencias de organismos internacionales, como se observa en la Tabla 1. Se programó la proteína manteniendo un aporte no mayor a 1,5 g por $\mathrm{kg}$ de peso, se controló el aporte y distribución de la grasa dada la prevalencia de dislipidemia en la población, al igual que los carbohidratos simples por la relación del exceso de su consumo con aumento de triglicéridos séricos y la aparición a futuro de Diabetes Mellitus (14).

Tabla 1. Fórmula Sintética planeada para menú de EPM

\begin{tabular}{|c|c|c|c|c|c|c|c|c|c|}
\hline Nutriente & Gramos & Kcal & $\%$ VCT & \multicolumn{6}{|c|}{ Calidad nutricional } \\
\hline \multirow[b]{2}{*}{ Proteína } & \multirow[b]{2}{*}{101,25} & \multirow[b]{2}{*}{405} & \multirow[b]{2}{*}{15} & \multicolumn{2}{|c|}{ Animal } & \multicolumn{4}{|c|}{ Vegetal } \\
\hline & & & & $\begin{array}{r}\% \\
70\end{array}$ & $\begin{array}{c}\mathrm{g} \\
70,9\end{array}$ & & $\begin{array}{r}\% \\
30\end{array}$ & $\begin{array}{r}9 \\
30\end{array}$ & \\
\hline \multirow[b]{2}{*}{ Grasa } & \multirow[b]{2}{*}{75} & \multirow[b]{2}{*}{675} & \multirow[b]{2}{*}{25} & \multicolumn{2}{|c|}{ Saturada } & \multicolumn{2}{|c|}{ Mono } & \multicolumn{2}{|c|}{ Poli } \\
\hline & & & & $\begin{array}{r}\% \\
7\end{array}$ & $\begin{array}{l}g \\
21\end{array}$ & $\begin{array}{l}\% \\
10\end{array}$ & $\begin{array}{l}g \\
30\end{array}$ & $\begin{array}{r}\% \\
8\end{array}$ & $\begin{array}{c}g \\
24\end{array}$ \\
\hline \multirow[b]{2}{*}{$\mathrm{CHO}$ `s } & \multirow[b]{2}{*}{405} & \multirow[b]{2}{*}{1620} & \multirow[b]{2}{*}{60} & \multicolumn{2}{|c|}{ Simples } & \multicolumn{3}{|c|}{ Complejos } & \\
\hline & & & & $\begin{array}{r}\% \\
10\end{array}$ & $\begin{array}{c}\mathrm{g} \\
67,5\end{array}$ & $\begin{array}{r}\% \\
50\end{array}$ & $\begin{array}{r}9 \\
33\end{array}$ & & \\
\hline Total & 2700 & 2700 & 100 & & & & & & \\
\hline
\end{tabular}

Teniendo en cuenta las condiciones de salud y riesgo nutricional de la población y los efectos en la salud de éstos micronutrientes, demostrados por diversos estudios, se vigilaron en la planeación de la minuta patrón y el menú el aporte especialmente de Vitaminas C y E , potasio, y fibra dietaría. Así mismo, se restringió el aporte de colesterol y sodio (15 - 27). 
Una vez definidas las recomendaciones nutricionales y su distribución de para la población atendida, se realizó un sondeo sobre gustos, rechazos, preferencias, hábitos alimentarios, y rasgos culturales para el diseño de la minuta patrón y el ciclo de menú en cada campamento. Igualmente se analizaron proveedores, productos disponibles en la región, equipos, planta física, y otros condicionantes para la preparación del menú, procurando evitar mayores alteraciones en el funcionamiento de los servicios.

La minuta patrón se planeó para desayuno, almuerzo y comida, con una distribución del valor calórico total -VCT- de $30 \%, 35 \%$ y $35 \%$ respectivamente. Se programó un menú cíclico partido para cinco semanas, en cada uno de los servicios de alimentación intervenidos. Se realizó el análisis nutricional de la alimentación suministrada en el programa MENÚ TIMÓN® el cual trabaja con la tabla de composición de alimentos colombianos del Centro de Atención Nutricional -CAN- (28) teniendo en cuenta algunos márgenes de error para micronutrientes, considerando la pérdida de calidad nutricional por métodos de cocción y preparación de los alimentos así como el tiempo transcurrido entre su preparación y consumo.

La implementación del nuevo menú requirió la adopción de diferentes medidas aplicadas progresivamente para dar cumplimiento a la planeación nutricional definida, tales como la modificación de algunos métodos de cocción utilizados: se redujeron las frituras; se sustituyeron productos de mesa; se comenzó a usar azúcar light y edulcorantes para diferentes preparaciones como reemplazo parcial de azúcar normal; se limitó el uso de enlatados y conservas. Se disminuyó en un $50 \%$ la adición de aceite y margarinas para las preparaciones y la frecuencia de oferta de alimentos altos en colesterol, y sodio. Por otro lado se aumentó: las preparaciones cocidas y asadas en el ciclo; el suministro de frutas; la oferta de derivados lácteos bajos en grasa; jugos de fruta natural; aguacate; y carnes blancas; y se incrementó la variedad en ensaladas incluyendo nuevos productos y combinaciones que favorecieran el aporte de micronutrientes a la dieta.

Análisis de datos. Los datos fueron procesados en SPSS $17.0^{\circledR}$ para su análisis estadístico. Se realizaron pruebas de normalidad y ajuste KolmogorovSmirnov, se aplicó la prueba t-Student pareada o Wilcoxon para muestras relacionadas en variables cuantitativas y prueba de cambios de McNemarBowker para variables de más de dos categorías. Con el fin de establecer la influencia de otras variables en los resultados se aplicaron modelos de regresión múltiple para $\mathrm{CT}$ y TG después de la intervención (CT2 y TG2) 
como variables dependientes; como variables independientes para ambos modelos se consideraron: tiempo de exposición a la intervención, edad, diferencia del IMC antes y después, frecuencia de actividad física, CT y TG iniciales (CT1 y TG1) y se comprobaron los supuestos de colinealidad, independencia (29) y normalidad de los residuales. Se consideraron como significativos valores de $\mathrm{p}<0,05$.

\section{RESULTADOS}

El $91,5 \%$ de la población evaluada fue de género masculino, con edad media de $45 \pm 8$ años, un promedio de $17 \pm 9,5$ años de servicio en la empresa, $13 \pm 8,9$ de ellos en campamentos.

Se encontró una prevalencia de sobrepeso y obesidad en el 75,2 \% de la población, determinada por IMC y un $91,5 \%$ de personas con exceso u obesidad por $\%$ de grasa. La frecuencia de clasificación por exceso de ambas variables antropométricas muestran un movimiento favorable posterior a la intervención especialmente en personas con obesidad grado 2 , reduciéndose de $23,3 \%$ a $19,4 \%$, y el porcentaje de personas clasificadas como Obesas por porcentaje de grasa pasó de $68,2 \%$ a $62,8 \%$ (Figuras 1 y 2).

Figura 1. Clasificación por IMC del personal evaluado antes y después de la intervención

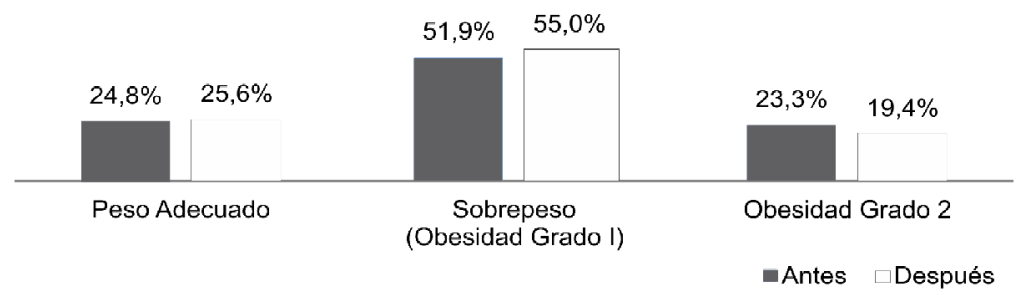

Pérdida promedio de $0,13 \pm 0,76$ unidades de IMC $p=0,063$

Al comparar el IMC y \% de grasa antes y después se encontró una pérdida promedio de $0,13 \pm 0,76$ unidades de IMC (IC $95 \%-0,23 ; 0,006)$ y $0,31 \pm 2,19 \%$ de grasa (IC $95 \%-0,69 ; 0,07$ ), sin que ello represente una disminución significativa ( $\mathrm{IMC} t=-1,88 ; \mathrm{p}=0,63 \mathrm{y} \%$ de Grasa $\mathrm{t}=-1,59 ; \mathrm{p}=0,11$ ) pero sí biológicamente plausible. 
Figura 2. Clasificación por Porcentaje de grasa del personal evaluado antes y después de la intervención

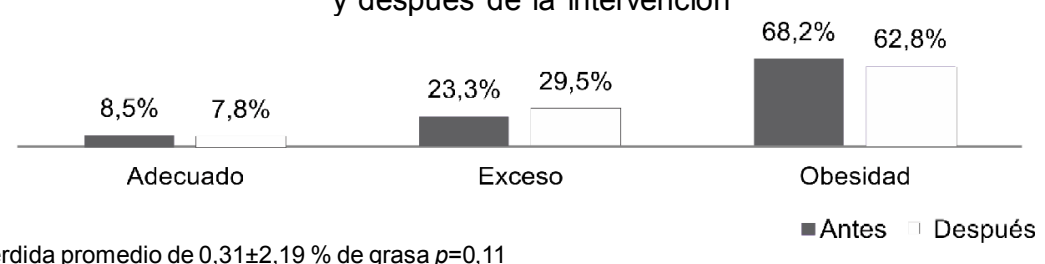

Pérdida promedio de $0,31 \pm 2,19 \%$ de grasa $p=0,11$

Las variables de perfil lipídico, mostraron una variación clínicamente favorable en todos los parámetros, especialmente en CT pasando del 34,9\% de personas con cifras de CT normal a 52,7 \% con una disminución media de 13,29 $\pm 36,4$ $\mathrm{mg} / \mathrm{dl}$ (IC $95 \%-214 ; 68$ ) y de TG normales de $27,9 \%$ a $51,9 \%$ con una reducción media de $81,19 \pm 138,1 \mathrm{mg} / \mathrm{dl}$ (IC $95 \%$-757; 81) (Figuras 3 y 4); al igual que se incrementó el porcentaje de personas con cHDL normal de 41,9\% a $48,1 \%$ con un incremento promedio de $3,29 \pm 8,1 \mathrm{mg} / \mathrm{dl}$ de cHDL (IC $95 \%$ $28,9 ; 29,1)$, sin embargo las personas con cifras normales de cLDL disminuyeron de $40,3 \%$ a 25,6 \% del total de la población evaluada, con un aumento promedio de 10,51 $\pm 30,6 \mathrm{mg} / \mathrm{dl}$ de cLDL (IC $95 \%$-91,1;95,4). Los cambios presentados en estos datos fueron significativos para todas las variables $(p<0,001)$.

Figura 3. Clasificación por niveles de colesterol total -CT- sérico, del personal evaluado antes y después de la intervención

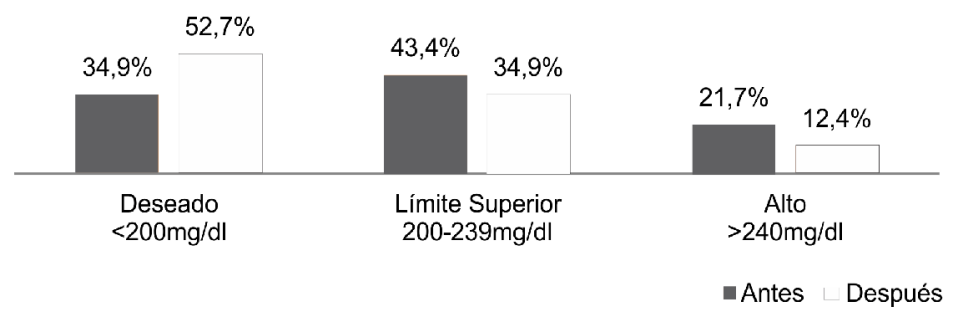

Pérdida promedio de 13,29 $\pm 36,4 \mathrm{mg} / \mathrm{dl}$ de CT $p=<0,001$

Figura 4. Clasificación por niveles de Triglicéridos -TG- séricos, del personal evaluado antes y después de la intervención

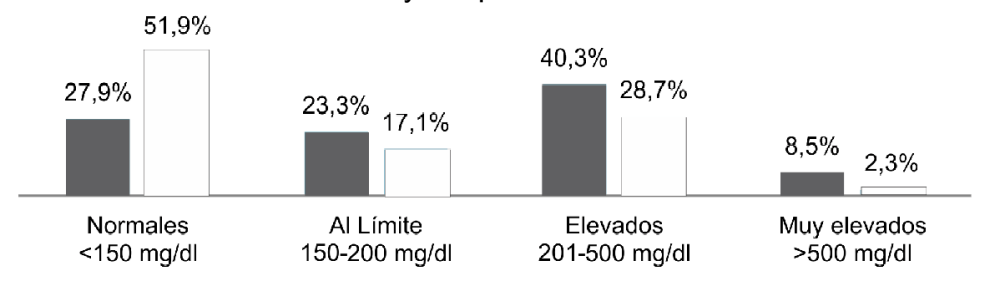

Pérdida promedio de $81,19 \pm 138,1 \mathrm{mg} / \mathrm{dl}$ de TG $p=<0,001$

- Antes $\sqcap$ Después 
$\mathrm{Al}$ correr los modelos de regresión para CT2 (modelo 1) y TG2 (modelo 2), se obtuvo que sólo la variable CT1 fue significativa en el modelo 1 y la variable TG1 en el modelo $2(p<0,001)$ lo cual demostró que el perfil lipídico no fue influenciado por otras variables no controladas como la actividad física, edad, tiempo de exposición y diferencias del IMC antes y después, por tanto se ratifica que el efecto en el perfil lipídico sí obedece a la intervención nutricional. Ambos modelos cumplieron con los criterios de colinealidad (FIV=-1,048 Modelo 1 y-1,015 Modelo 2) independencia (DW=1,97 Modelo 1 y 1,90 Modelo 2) y normalidad de residuos $(\mathrm{p}<0,001)$.

\section{DISCUSIÓN}

Los resultados en relación a las medidas antropométricas para clasificación del estado nutricional son relevantes en cuanto se evidencia una reducción en las personas clasificadas con exceso por todos los parámetros, y a pesar de no ser significativas estadísticamente, como lo demuestran los resultados, se destaca la pérdida depeso, IMC y porcentaje de grasa durante el corto período de intervención, en comparación con estudios de pérdida de peso y modificación de la dieta (30-32). Aunque los porcentajes de reducción global son bajos sí se encuentra que una alta proporción de la población bajó de peso (62,9\%) o se mantuvo (9\%), lo cual indica que la intervención tuvo efectos biológicamente plausibles en tanto se relaciona con la disminución de riesgos de salud, donde se destacan casos individuales con mejorías que representan ganancias notables para su salud.

Estos resultados llaman la atención dado que la intervención se concentró en la alimentación ofrecida en el servicio de alimentación de los campamentos sin modificar la recomendación de calorías para la población ni las porciones servidas en la cantidad esperada, sin embargo es posible que sólo el control de cantidad y calidad de las grasas y azúcares concentrados adicionados a las preparaciones haya contribuido con este desenlace (33-35). Es de anotar que el estudio tampoco controló el consumo de alimentos por fuera del servicio ni en los espacios de descanso de la población y que ésta mantuvo durante la intervención su rutina laboral, social y de actividad física, lo que demuestra que este tipo de intervenciones pueden ser realmente efectivas para la promoción de la salud y la prevención de la enfermedad, especialmente para aquellas patologías relacionadas con hábitos alimentarios inadecuados.

En cuanto al perfil lipídico, los resultados muestran un comportamiento de reducción altamente significativos en especial CT y TG, lo cual es coherente con los resultados hallados en otras investigaciones donde se demuestra la eficiencia de alimentos funcionales en el manejo dietético de pacientes con dislipidemia y 
obesidad en períodos de tiempo incluso más reducidos (17). Esto además se puede atribuir a las modificaciones hechas al menú en la disminución de azúcares concentrados, control de grasas e inclusión de alimentos altos en fibra en los servicios ofrecidos.

De los resultados emerge otra arista para el análisis, y se refiere específicamente a las variaciones encontradas en los niveles de cLDL que se incrementaron para la segunda medición con respecto a la inicial, esto fue reportado también por Pelletier y Col. (35) en un estudio antes y después de someter a un grupo de hombres con obesidad abdominal con edades entre 30 y 65 años a cambios en el estilo de vida, concluyendo que dicho incremento en cLDL podría estar relacionado con el aumento del cHDL y la reducción de TG. No obstante este aspecto podría ser objeto de análisis central en estudios posteriores con mayor tiempo de intervención (35-37). Ahora, es posible que la no disminución de cLDL pudiera también ser consecuente con la resistencia de los usuarios al consumo de alimentos integrales y en general de fibra dietética que se relaciona con la reducción de estos parámetros según diversos estudios $(24,25)$, porque aunque se ha incluido paulatinamente en su dieta, su consumo aún no es consistente, pero sería prematuro concluir sobre este aspecto.

Con respecto a la fracción de colesterol cHDL se destaca su incremento como positivo dado que no se modificó la actividad física de los individuos, por tanto su aumento se atribuye sólo a la dieta pues es coherente con la inclusión de nueces, semillas, aguacate y fuentes de ácidos grasos mono saturados en la alimentación, que se asocia con dicho resultado en diferentes estudios reportados en el mundo (21-23).

Después de analizar el posible efecto de las variables de confusión (diferencias del IMC antes y después, frecuencia de actividad física, edad, tiempo de exposición a la intervención), en el cambio del perfil lipídico, se comprueba que ninguna de ellas se relacionó directamente con éste y por tanto se puede atribuir el resultado sólo a la modificación de la alimentación.

Las principales limitaciones del estudio fueron la cultura alimentaria de los usuarios, su forma de agremiación laboral y la ubicación geográfica de los campamentos intervenidos, muy distantes unos de otros. En los servicios de alimentación se dificultó la intervención por contar con personal empírico y móvil, tener diferentes tipos de administración de un campamento a otro, y la constante necesidad de atender eventos especiales de la empresa.

El estudio ratifica la necesidad de generación de conocimiento a partir de nuevas investigaciones en el área de la nutrición en el mundo del trabajo, aspecto 
que actualmente es pobre en Colombia y que podría en el mediano y largo plazo, servir de referente en la creación de políticas claras en relación con la protección y promoción de la salud de la población trabajadora del país, comprometiendo al sector público pero también al sector privado •

Agradecimientos: Las autoras agradecen a Empresas Públicas de Medellín, en especial a la Subgerencia de Generación de Energía, al personal administrativo yoperativo de los diferentes campamentos, y servicios de alimentación, a la Empresa Regional de Servicios -ERS-, los estudiantes de práctica, las nutricionistas participantes, la Escuela de Nutrición y Dietética de la Universidad de Antioquia, y principalmente a nuestros esposos e hijos, por el apoyo recibido de cada uno en la construcción de este proceso que hoy satisfactoriamente concluimos.

\section{REFERENCIAS}

1. Organización Mundial de la Salud -OMS-. Informe sobre la salud en el mundo 2008. [Internet]. Disponible en: http://www.who.int/whr/2008/es/ Consultado Julio del 2010.

2. Wanjek C. Food at Work: Workplace solutions for malnutrition, obesity and chronic diseases. International Labour Office Geneva; 2005.

3. Instituto Colombiano de Bienestar Familiar ICBF. Encuesta Nacional de la situación nutricional en Colombia, 2005. Santafé de Bogotá; Colombia; 2006. p. 83-85.

4. Colombia. Congreso de la República. Ley 1355 de Octubre de 2009. Por la cual se define la obesidad y las enfermedades crónicas no transmisibles asociadas a ésta como una prioridad de salud pública y se adoptan medidas para su control, atención y prevención. Bogotá; 2009.

5. Colombia. Ministerio de la Protección Social. Resolución número 003673 de 2008. Por la cual se establece el reglamento técnico de trabajo seguro en Alturas. Bogotá; 2008.

6. De Irala J, Martínez MA, Seguí M. Epidemiología Aplicada. Ariel Ciencias Médicas. Segunda Edición actualizada. Barcelona; 2008.

7. Restrepo, MT. Manual de normas técnicas para la toma de medidas antropométricas. Universidad de Antioquia. Medellín; 2004.

8. Durnin, JVA, Womersley J. Body fat assessed from total body density and its estimation from skin fold thickness: measurements on 481 men and women aged from 16 to 72 years. Br. J. Nutr. 1974; 32: 77-97.

9. Consenso SEEDO 2000 para la evaluación del sobrepeso y la obesidad y el establecimiento de criterios de intervención terapéutica. Sociedad Española para el estudio de la obesidad. Med Clin. Barcelona 2000; 115: 589-597.

10. Chaves A, Vargas M, Schosinsky K. Evaluación de método enzimático colorimétrico para la cuantificación de colesterol sérico. Rev. Costarric. Cienc. Méd. jul. 1997; 18(1): 30-43.

11. Díaz J. 770 Preguntas y respuestas: Determinaciones Bioquímicas, utilidad y valoración clínica. Editorial Masson S.A. Barcelona; 2004.

12. NCEP. Detection, evaluation and treatment of high blood cholesterol in adults (Adult treatment Panel III) [Internet] 2002. Disponible en: http://www.nhlbi.nih.gov/about/ncep/index.htm. Consultado junio del 2010.

13. Instituto Colombiano de Bienestar Familiar -ICBF-. Recomendaciones de consumo de Calorías y Nutrientes para la Población Colombiana. Bogotá; 1988.

14. Colombia. Ministerio de la Protección Social. Guías Alimentarias para la Población Colombiana mayor de 2 años. Bases técnicas. Bogotá; 2004.

15. Howard B, Van L, Hsia J. Low fat dietary pattern and risk of cardiovascular disease: The women`s health initiative Randomized controlled dietary modifications trial. JAMA 2006; 295: 655-666. 
16. Lichtenstein A, Ausman L, Jalbert SM, Vilella M, Jauhiainen M, McGladdery S, et al. Efficacy of a therapeutic lifestyle change step 2 diet in moderate hypercholesterolemic middle - aged and elderly female and male subjects. J Lipid Res 2001; 43: 264-273.

17. Menzink R. Effects of dietary fatty acids and carbohydrates on the ratium of serum total to HDL cholesterol and on serum lipids and apolipoproteins: A meta-analysis of 60 controlled trials. Am J Clin Nutr 2003; 77: 1146-1155.

18. Kris $\mathrm{P}$, William $\mathrm{H}$, Lawrence J. Fish consumption, fish oil, omega 3 fatty acids and cardiovascular disease. Circulation. 2002;106:2747.

19. Davis B, Kris P. Achieving optimal essential fatty acid status in vegetarians: current knowledge and practical implications. Am J Clin Nutr 2003; 78: 640S-646S.

20. Hu FB, Stampfer MJ, Manson JE, Rimm EB, Wolk A, Colditz GA, et al. Dietary intake of alpha linolenic acid and risk of fatal ischemic heart disease among women. Am J Clin Nutr. 1999; 69: 890 - 897.

21. Kris PM, Zhao G, Binkoski AE, Coval SM, Etherton TD. The effects of nuts on coronary heart disease risk. Nutr Rev 2001; 59: 103 - 111.

22. Pieterse Z, Jerling J, Oosthuizen W. Avocados, monounsaturated fatty acids, weight loss and serum lipids. Specialist Forum 2003; 3: 65-72.

23. Díaz M, Toro $\mathrm{C}$. Efecto de la adición de aguacate a la alimentación habitual sobre los niveles de lípidos de personas con dislipidemia. Universitas Scientiarum. 2004; 9: 49-58.

24. Jenkins DJ, Kendall CW, Vuksan V, Vidgen E, Parker T, Faulkner D, et al. Soluble fiber intake at dose approved by the US FDA for a claim of health benefits: serum lipid risk factors for cardiovascular disease assessed in a randomized controlled crossover trial. Am J Clin Nutr 2002; 75: 834-839.

25. Pereira MA, O'Reilly E, Augustsson K, Fraser GE, Goldbourt U, Heitmann BL, et al. Dietary fiber and risk of coronary heart disease: a pooled analysis of cohort studies. Arch Intern Med. 2004; 164: 370-376.

26. Patel M, Thompson P. Phytosteroles and cardiovascular disease. Atherosclerosis 2006; 186: 12-19.

27. Gray J, Griffin B. Eggs and dietary cholesterol dispelling the myth. Nutrition Bulletin. 2009; 34: 66-70.

28. Quintero D, Alzate MC, Moreno S. Tabla de Composición de Alimentos. Segunda Edición. Centro de Atención Nutricional. Medellín; 2003.

29. Freund R, Wilson W. Regression Analysis: Statical modeling of response variable. Academic Press. United States of America; 1998.

30. Carneiro FM, Bosch V, Izquierdo M. Efectos de la intervención nutricional sobre las variables antropométricas, la ingesta y las concentraciones de lípidos y lipoproteínas del plasma en niños con dislipidemia. ALAN; 2001; 51: 132-144.

31. Moreno GM, Manrique M, Guzmán S, Maiz A, Patiño C, Valdés R, et al. Cambios en los factores de riesgo metabólicos en pacientes obesos en tratamiento. Rev. méd. Chile 2000; 128(2): 193-200.

32. Basulto J, Bultó L, Chamorro M, Lafuente C, Martín E, Porta G. Análisis de programa de pérdida de peso con sustitutivos de comidas sobre el control del peso y parámetros bioquímicos en pacientes con sobrepeso y obesidad grado I Nutr Hos. 2008; 23(4):388-394.

33. Pelletier E, Arsenault B, Lemieux I, Alméras N, Bergeron J, Tremblay A, et al. Correlates of Changes in HDL and LDL Particle Size in Response to a Physical Activity/Eating Lifestyle Modification Program in Viscerally Obese Men. Circulation. 2009;120:S482.

34. LeCheminant J, Smith B, WestmanE, Vernon M, Donnelly J. Comparison of a reduced carbohydrate and reduced fat diet for LDL, HDL, and VLDL subclasses during 9-months of weight maintenance subsequent to weight loss. Lipids in Health and Disease 2010; 9-54.

35. Nicholls SJ, Tuzcu EM, Sipahi I, Grasso AW, Schoenhagen P, Hu T, et al. Statins, high-density lipoprotein cholesterol, and regression of coronary atherosclerosis. JAMA 2007; 297(5):499-508. 